\title{
Closure and Telicity across Categories
}

\author{
Yoad Winter \\ Technion
}

\section{Introduction}

One of the fascinating aspects of telicity is its relations with non-verbal categories. Whether a given predicate is telic or atelic does not only depend on the verb, but can also be affected by the count/mass quantification in the NP object, by the lexical semantics of related adjectives, and by the prepositions that the predicate may contain. The latter two factors are in the focus of this paper. For example, the telicity of the deadjectival verb straighten and the complex verb phrase jump into the water, is related to the semantic structures of the adjective straight or the preposition into. A mathematical notion that is relevant to semantic domains across different categories is the notion of closed intervals - intervals that include their limit points. This paper studies the relations that closure properties of adjectives and prepositions bear to the (a)telicity properties of verb phrases. Following (Rotstein and Winter 2004), closure of adjectival scales is treated as the defining distinction between two kinds of adjectives (e.g. straight vs. bent) that affects their behavior with almost modifiers. This leads to a conception of closure as the key source of ambiguity in verb phrases modified by almost, which is opposed to Dowty's (1979) characterization of such ambiguities in terms of telicity. The telic/atelic distinction between predicates is made explicit and is related to closure of adjectival scales and prepositional paths. This is done using a new notion of weak downward monotonicity, which refines the simple but crude subinterval (homogeneity) characterization of atelic predicates that was proposed in (Bennett and Partee 2003).

Throughout this paper, when I say that a predicate is telic, I first of all mean that it does not support entailments from its progressive VP forms to corresponding non-progressive forms. Thus, telic/atelic classifications are supported by contrastive pairs like the following.

(1) Mary was drawing a circle $\not \Rightarrow$ Mary drew a circle (telic)

(2) Mary was pushing a cart $\Rightarrow$ Mary pushed a cart (atelic)

Many other syntactic and semantic effects have been classified as telicity phenomena. Especially, acceptability with for/in time adverbials is often used as another central test for a/telicity. However, since the correspondence between different tests

Special thanks to Carmen Rotstein, whose insights on deadjectival verbs prompted me to work on this topic. Thanks also to James Higginbotham, Malka Rappaport Hovav, Chris Kennedy, Stephen Wechsler, Joost Zwarts and to the audiences at SALT 16 (Tokyo 2006) and at the workshop on scalar meaning (Chicago 2006). Chris Kennedy's invitation to the workshop in Chicago was a valuable encouragement for me while working on this paper. An extended version can be found at my webpage: http://www.cs.technion.ac.il/ 〜winter 
for telicity is not complete, I will use entailment tests as in (1) and (2) as the primary test that is relevant for the phenomena treated in this paper.

Telicity factors interact with another cross-categorial phenomenon - constraints on the acceptability of the modifier almost. Addressing these relations, (Dowty 1979) discusses two possible interpretations of sentences like the following, where almost combines with a telic predicate.

(3) Dan almost drew a circle.

One type of interpretation, which following (Rapp and von Stechow 1999) I call the scalar (S) interpretation of almost, states that Dan started to draw a circle and got close to finishing it. Another kind of interpretation allows (3) to be true if Dan only got close to starting a drawing of a circle but didn't actually start. Rapp and von Stechow dub this the counterfactual (C) interpretation of almost. In many cases the S-interpretation of almost is not present. Consider for instance the following example, where almost modifies an atelic predicate.

(4) Dan almost pushed a cart.

In (4), the only possible interpretation of almost is the C-interpretation. An Sinterpretation, claiming that Dan started and almost finished to push a cart, is hardly available. Dowty uses the availability of an S-interpretation for almost as a characteristic of telic predicates. Let us state this as in the following hypothesis.

(5) Scalarity-telicity hypothesis: A scalar interpretation of almost is available with a predicate $\mathrm{P}$ if and only if $\mathrm{P}$ is telic.

In view of the cross-categorial behavior of almost and the increasing body of work in recent years on scale structures of adjectives and path structures of prepositions, the value of Dowty's hypothesis for current research is in making another possible connection between such non-temporal ontologies and telicity.

This paper studies this connection further while taking Dowty's hypothesis as a starting point. My plan is as follows. In Section 2 I will argue that Dowty's hypothesis as stated in (5) is not completely adequate, and that the factor that affect S-interpretations is not telicity per se. Rather, I will claim that the same closure requirement that according to (Rotstein and Winter 2004) determines acceptability of almost with adjectives should be used to describe the availability of its S-interpretation with verbs. Section 3 develops this idea by concentrating on the empirical relations between the scale structure of an adjective and the (a)telicity of the corresponding deadjectival verb. Section 4 formalizes the mapping from adjectives to deadjectival verbs and refines the subinterval property of atelic predicates that was proposed by (Bennett and Partee 2003). With this novel characteristic of atelic predicates, which is called weak downward monotonicity, it is shown that an open/closed scale structure of an adjective directly leads to an a/telic temporal structure (respectively) of the corresponding verb. Section 5 shows that this is no longer the case with respect to the more complex relations between the path structures of directional prepositions and the (a)telicity of verb phrases containing them. This dissociation between closure and telicity with directional PPs accounts for the counterexamples to Dowty's scalarity-telicity hypothesis. 


\section{Almost and Prepositions: Dissociating Telicity from closure}

(Zwarts 2005) discusses some systematic differences between directional prepositions in terms of telicity. Using the in/for adverbial test, Zwarts addresses contrasts like the following.

(6) a. Alex ran toward the park/along the river *in/for a day.

b. Alex jumped into the water/ran to the park in/*for ten minutes.

c. Alex ran around the lake/across the bridge/through the tunnel in/for one hour.

Zwarts' classification of prepositions is accordingly:
Atelic: $\quad$ toward, along
Telic: $\quad$ to, into, onto, from, out of, off, away from, past, via
Telic/atelic: around, across, down, over, through, up

This classification agrees with the following judgements on the validity of simple entailments from the past progressive to the past simple.

(8) Dan was running toward the park $\Rightarrow$ Dan ran toward the park

Dan was running along the river $\Rightarrow$ Dan ran along the river

(9) Dan was running to the park $\nRightarrow$ Dan ran to the park

Dan was jumping into the water $\nRightarrow$ Dan jumped into the water

(10) Dan was running around the lake $\not \Rightarrow$ Dan ran around the lake

Dan was running across the bridge $\nRightarrow$ Dan ran across the bridge

Dan was running through the tunnel $\nRightarrow$ Dan ran through the tunnel

Note that we should expect that any telic reading of prepositions like around, across and through would block entailments as in (10) even if, as Zwarts proposes, these prepositions are ambiguous between telic and atelic interpretations.

The lack of entailment in (9) and (10) may lead us to expect, according to Dowty's hypothesis in (5), that the prepositions in these sentences support the S-interpretation of almost. However, there are some systematic contrasts that cast doubts on this expectation. Consider first the contrast between (11a) and (11b).

(11) a. Dan almost walked around the lake. (C/?S)

b. Dan almost circled the lake. $(\mathrm{C} / \mathrm{S})$

While it is easy for native speakers to accept (11b) as true if Dan started a circle around the lake but didn't finish it, judgements are less clear in (11a). The Cinterpretation is possible in both (11a) and (11b), meaning that John was close to starting a path around the lake, but actually did not. A similar contrast appears between the following sentences.

(12) a. Dan almost walked across the bridge. (C/?S)

b. Dan almost crossed the bridge. $(\mathrm{C} / \mathrm{S})$ 
Infelicity of S-interpretations with telic prepositions also appears in the following examples.

(13) Dan almost went to the park. (C/?S)

(14) Dan almost drove through the tunnel. (C/?S)

Quite in accordance with the marginality of these S-interpretations, English speakers I consulted had difficulties in accepting sentences like the following, where almost is prefixed to the PP itself.

(15) ?Dan ran almost around the lake/across the bridge/to the park.

While judgements are sometimes insecure on such English sentences, there are other languages that allow us to draw a clear distinction between telic prepositions. Modern Hebrew is one language where a preposition parallel to English until also has spatial usages. The Hebrew preposition @ad (='until') shows a clear contrast with the preposition le/la (='to/to-the') in terms of allowing modification using kim@at (='almost'). Consider the following Hebrew sentences.

a. ? dan rackim@at la'agam.

Dan ran almost to-the-lake

'Dan ran almost to the lake' (?S)

b.danrackim@at@ad ha'agam.

Dan ran almost until the-lake

'Dan ran and almost reached the lake' (S)

Hebrew speakers react to sentence (16a), with the preposition le, in a similar hesitation to English speakers who are confronted with sentences like (15). By contrast, sentence (16b) with the preposition @ ad is clearly acceptable and, as evident from the translation, supports an S-interpretation for the modifier kim@at (='almost').

As can be expected, when kim@at appears before the VP, a noticeable distinction in the availability of the S-interpretation shows up between le and @ad:

a.dan kim@at rac la'agam.

Dan almost ran to-the-lake

'Dan almost ran to the lake' $(\mathrm{C} /$ ?S $)$

b.dan kim@at rac@ad ha'agam.

Dan almost ran until the-lake

Ambiguous (C/S) -

C: 'Dan almost ran to the lake'

S: 'Dan ran and almost reached the lake'

A similar contrast can be observed in Dutch (Joost Zwarts, p.c.), with the prepositions naar (='to') and tot (='until'):

a. Dan rende bijna naar het meer.

Dan ran almost to the lake

'Dan almost ran to the lake' (C) 
b. Dan rende bijna tot (aan) het meer.

Dan ran almost until the lake

'Dan ran and almost reached the lake' (S)

Why do pairs of telic predicates show contrasts in their tolerance toward the S-interpretation of almost modification, as opposed to what we might expect from Dowty's hypothesis in (5)? My proposed answer essentially follows Rotstein and Winter's intuition regarding the acceptability of almost modification. Rotstein and Winter propose that for almost modification of adjectives to be felicitous, the scale that is modified has to be closed - it should have a maximal point within it. Below I will extend this requirement for spatial and temporal-aspectual structures. ${ }^{1}$ I will argue that this closure condition by itself is sufficient for telicity, but it is not a necessary condition for telicity. Thus, telic structures may be open, and hence may disallow S-interpretations of almost.

Consider Figure 1. I propose that the path of spatial movement that we associate with prepositions like Hebrew le, Dutch naar or English to is open. By contrast, the corresponding path with @ad/tot (='until') is minimally different but closed. Similarly, for the preposition around the path is proposed to be open, whereas the verb to circle is required to have a closed path. This difference is proposed as the factor that controls differences in acceptability with almost, but all the paths that are described in Figure 1 will be formally characterized as telic.
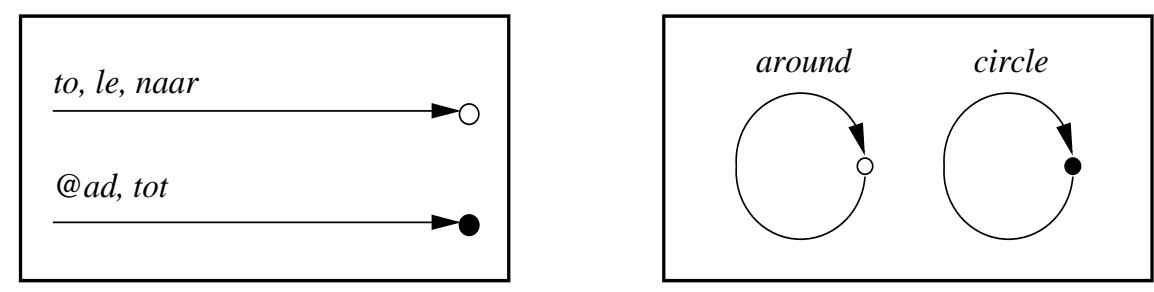

Figure 1: telic paths - open and closed

This line of explanation leaves us with some points that should be addressed.

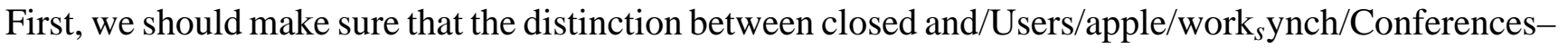
Talks/2006/salt06/SALT Source Files/tex/Winter/winter-SALT16-rev/winter-SALT16.tex open adjectival scales carries over to the verbal domain. Important aspects of this mapping were already studied in (Hay et al. 1999), but almost modification provides us with an additional test for closure and allows to define the mapping from scales to temporal structures more explicitly. Second, we should be able to formally derive our hypothesis that closed structures are inherently telic. Third, something similar should be done with the mapping from path structures of prepositions to the temporal-aspectual domain where telicity is manifested. These issues are addressed in the following sections.

\footnotetext{
${ }^{1}$ For a similar parallelism between adjectives and verbs see (Amaral 2006).
} 


\section{3. 'Almost', Adjectives and the Telicity of Deadjectival Verbs}

This section first reviews Rotstein and Winter's treatment of almost modification with adjectives of different scale structures. Then we move on to using this proposal in characterizing the (a)telicity of deadjectival verbs.

\section{1. 'Almost' and the Total/Partial Distinction}

Previous works (Cruse 1986, Yoon 1996, Kennedy and McNally 2005, Rotstein and Winter 2004) study pairs of adjectives like dry-moist, clean-dirty, and healthysick, which Yoon calls total and partial adjectives, respectively. Intuitively, one contrast between such adjectives is that partial adjectives indicate some amount of the relevant property (moisture, dirt, sickness etc.), whereas the total adjectives indicate no amount of this property. For instance, a dirty object has some degree of dirt, but it is not necessarily free of cleanliness. Conversely, a clean object is free of dirtiness, and it would be too weak to describe it as only having some degree of cleanliness. Some other pairs of adjectives where such intuitive distinctions hold are given in (19).

(19) Total-partial adjectives: dry-moist, clean-dirty, healthy-sick, straightbent/curved, smooth-rough, complete-incomplete, sharp-unsharp/blurred

The distinction between total and partial adjectives was recently corroborated by (Frazier et al. 2006) in a series of psycholinguistic experiments. Following (Kennedy 2005), other pairs of antonymous adjectives will henceforth be referred to as relative. $^{2}$ Some typical examples for relative adjectives follow.

(20) Relative adjectives: long-short, expensive-cheap, wide-narrow, high-low, big-small, happy-unhappy etc.

Cruse, and more recently Rotstein and Winter, study the different behavior of almost with total, partial and relative adjectives. In out-of-the-blue contexts, a modified construction almost $A$ is perfectly $\mathrm{OK}$ when $A$ is a total adjective. But almost $A$ is often marginal when $A$ is partial or relative. Consider for instance the following examples.

(21) almost dry/?moist, almost clean/?dirty, almost straight/?bent, almost smooth/ ?rough, almost complete/?incomplete

(22) ?almost long/short, ?almost expensive/cheap, ?almost wide/narrow, ?almost big/small, ?almost fast/slow

Rotstein and Winter (henceforth $\mathrm{R} \& \mathrm{~W}$ ) point out that with some total-partial pairs of adjectives this contrast in acceptability is not so sharp. For instance, according to our intuitive criterion, the adjectives healthy and sick should be classified as a

\footnotetext{
${ }^{2}$ Kennedy and McNally refer to total and partial adjectives as maximum/minimum standard adjectives. (Kennedy 2005) further refers to the adjectives in these two subclasses as absolute, in distinction to the relative adjectives.
} 
total-partial pair, but almost sick is not worse than almost healthy. $\mathrm{R} \& \mathrm{~W}$ therefore suggest a simple semantic test that more easily identifies the almost contrast in totalpartial pairs of adjectives. For instance, for the adjectives healthy and sick R\&W note that if someone is almost sick, she is necessarily not healthy. By contrast, an almost healthy person can be (at least slightly) sick. This is illustrated below.

(23) \#She is almost sick but (still) healthy.

(24) She is almost healthy but (still) sick.

Similar contrasts can be shown for the other adjectives in (19).

It was pointed out by Chris Kennedy (p.c. and in Kennedy 2005) that both partial adjectives and relative adjectives may allow almost in contexts that explicitly specify a standard. Some such examples are given below.

(25) We consider a glass dirty and wash it as soon as there are five spots on it. This glass is now almost dirty - it has four spots on it.

(26) The publisher considers a book long if it's 300 pages or more. This book is almost long - it's 298 pages.

To deal with these different behaviors of total, partial and relative adjectives, $\mathrm{R} \& \mathrm{~W}$ propose three kinds of scale structures:

1. Total scales, in which the standard value is fixed on the zero degree and creates a closed interval of degrees associated with the adjective.

2. Partial scales, where the standard value is set by default to the zero degree and creates an open interval. This default can be overridden by context, which may then fix a standard at a positive degree and create a closed interval.

3. Relative scales are like partial scales, but they lack a default standard. Without a context, their standard can be anywhere and creates an open interval.

These three types of scales, with the possible contextual effects on them, are illustrated in Figure $2 .{ }^{3} \mathrm{R} \& \mathrm{~W}$ 's semantics of almost assumes that this modifier requires that the interval $I$ of degrees associated with an adjective $A$ on the scale is closed from below. The interval associated with the adjectival phrase almost $A$ is then assumed to be a (short) open interval adjacent to $I$. This requirement accounts for the facts observed above, and is illustrated in Figure 3. Throughout this paper I adopt this theoretical arrangement, which $\mathrm{R} \& \mathrm{~W}$ discuss at length in their paper.

\subsection{The Telicity of Deadjectival Verbs}

One of the central topics in works on aspect and telicity has been to characterize the telicity of what (Dowty 1979) calls degree achievement verbs: verbs like grow, fall, increase, smoothen etc. Verbs that are morphologically and semantically related

\footnotetext{
${ }^{3}$ See also (Kennedy and McNally 2005, Hay et al. 1999, Kennedy 2005) for similar distinctions and further discussion.
} 


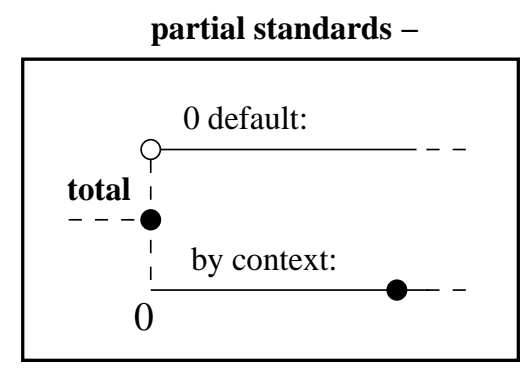

Figure 2: total, partial and relative scales
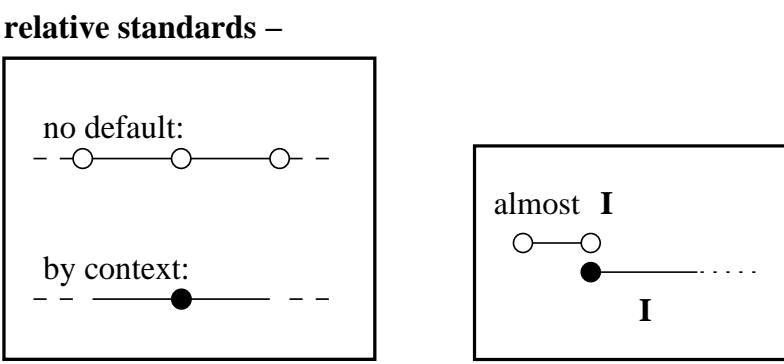

Figure 3: almost

to adjectives constitute an important subclass of the 'degree achievement' verbs. I henceforth refer to such verbs as deadjectival (DA) verbs. Some examples of DA verbs related to total, partial and relative adjectives are given below.

Total-partial DA verbs: dry-moisten, clean-dirty, straighten-bend/curve, smoothen-roughen, sharpen-unsharpen/blur

(28) Relative DA verbs: lengthen-shorten, widen-narrow, heighten-lower

As many works point out, ${ }^{4}$ there are strong relations between (a)telicity of DA verbs and the classification of the corresponding adjectives. For instance, consider a sentence with a total DA verbs like the towel was drying. This sentence clearly does not entail that the towel dried: the drying process might have stopped in the middle. By contrast, the sentence the towel was moistening more likely entails that the towel moistened, at least with the 'default' understanding of moist. A similar 'default' reasoning appears with relative DA verbs:
a. Jane was lengthening the rope $\stackrel{D}{\Rightarrow}$ Jane lengthened the rope
b. Jane was shortening the rope $\stackrel{D}{\Rightarrow}$ Jane shortened the rope

Hay et al. (1999) show that world knowledge or contextual factors may block such default conclusions with relative adjectives. Consider their examples below.

(30) The tailor was lengthening my pants $\nRightarrow$ The tailor lengthened my pants

(31) Kim was lowering the blind $\nRightarrow$ Kim lowered the blind

Clearly, lengthen in (30) requires that a certain length be attained, hence the failure of the inference. Likewise for (31). Something similar can be shown with partial DA verbs. Consider a computer program that runs over every pixel in a photo, and may change its color so to produce a 'romantic' blurred atmosphere in the picture. If we stop the program at the middle of the blurring process, it does not follow that the program blurred the photo. Obviously, there is a certain level of blurring that should be attained for this to happen. Thus we conclude that despite the out-of-the-blue atelicity of partial DA verbs like blur or unsharpen, they do not support atelicity entailments when a non-zero standard is assumed as in the following example.

\footnotetext{
${ }^{4}$ See (Declerck 1979, Hay et al. 1999, Kearns 2005), among others.
} 
(32) The program was blurring/unsharpening the photo $\nRightarrow$ The program blurred/ unsharpened the photo

We see that the atelicity of partial and relative DA verbs is only a tendency, which is sensitive to contextual factors. By contrast, as far as I could check it this is not the case with the telicity of total DA verbs. I find it hard to think of contexts that allow to conclude that the towel dried when all we know is that the towel was drying. Similarly for other total DA verbs: all of them seem to behave in this respect like typical telic predicates. ${ }^{5}$ We summarize this in the following generalization.

(G1) Total DA verbs are uniformly telic. Partial and relative DA verbs are atelic by default, but contextual factors can make them telic.

From the literature on DA verbs we can also extract a related generalization concerning the relations between the DA verb and the positive form of the corresponding adjective. Consider the following sentences.

a. Mary has dried the towel/cleaned the room/straightened the rope.

b. Mary has moistened the towel/dirtied the room/bent the rope.

a. Jane has lengthened the rope/widened the gap/heightened the book.

b. Jane has shortened the rope/narrowed the gap/lowered the book.

From the respective examples in both (33a) and (33b) we can conclude that the towel is dry/moist, that the room is clean/dirty, etc. By contrast, from (34a) and (34b) we cannot conclude that the rope is long/short, that the gap is wide/narrow etc. Thus, with both total and partial DA verbs, the present perfect tense entails the positive form of the adjective. However, with relative DA verbs this is not the case. Even in cases like Hay et al.'s (30) and (31), where the standard that is assumed forces the relative DA verb to behave in a telic manner, the positive form of the adjective is not entailed. Consider the following lack of entailments.

(35) The tailor has lengthened my pants $\nRightarrow$ My pants are long

(36) Kim has lowered the blind $\nRightarrow$ The blind is low

Concluding, I suggest the following generalization.

(G2) Total DA verbs and partial DA verbs entail the positive form of the respective adjective, whereas relative DA verbs do not.

Let us now move on to the account of generalizations (G1) and (G2).

\section{The A-V Mapping and Telicity}

To account for the facts that are summarized under (G1) and (G2) we need to have a mechanism that systematically relates the meaning of DA verbs to the meaning

\footnotetext{
${ }^{5}$ Note, however, that many total DA verbs allow modification by for adverbials, as in Declerck's (1979) example: the corn dried for three days. However, as (Dowty 1979) points out, for adverbials can turn a (telic) accomplishment into an (atelic) activity, so they cannot always be relied on as a test for atelicity. See more on other telicity tests for such verbs in (Hay et al. 1999, Kearns 2005).
} 
of the corresponding adjectives. Further, to account for generalization (G1) we have to use a formal characteristic of telicity that distinguishes between the three classes of DA verbs in accordance with their temporal-aspectual behavior. These two problems are the subject of this section.

\subsection{The A-V Mapping}

We will assume that verbs have a temporal interval argument that is responsible for describing their temporal behavior. ${ }^{6}$ DA verbs describe a process that is related to a degree change of one of their arguments - subject in intransitive sentences (e.g. the gap widened), object in most transitive sentences (e.g. the workers widened the road). We assume that a DA verb holds at the temporal intervals $I$ that satisfy the following two properties, in relation to the relevant argument:

(37) Progress - the degree of the verb's argument on the scale of the corresponding adjective increases during the interval $I$.

(38) Culmination - at the end of the interval $I$, the degree attained is within the set of degrees associated with the adjective, if such a set is specified by a given standard value.

Consider for example the total DA verb straighten. 'Progress' means that at any temporal interval $I$, an entity $x$ straightens at $I$ only if at the end of $I$ the entity $x$ is straighter than what it was at the beginning of $I$. 'Culmination' means that at the end of $I$ the entity $x$ is within the set of degrees associated with straight, i.e. the sentence $x$ is straight is true. By our definition of total scales this means that a straightening process entails moving from a positive degree of curvedness to zero curvedness. For a partial DA verb like bend, progress and culmination entail that without contextual information, a bending process involves moving from any degree of curvedness to a higher and positive degree of curvedness. For a relative DA verb like widen, in the absence of contextual information a widening process only requires progress (i.e. becoming wider). This is because culmination would require there to be a standard value associated with the adjective wide, and no such standard is given by default. These three different kinds of process that we assume are illustrated in Figure 4.

It is easy to see how these assumptions account for generalization (G2) in the absence of contextual knowledge: total and partial adjectives have a default standard, hence culmination is responsible for the entailment from the DA verb to the positive form of the adjective. Conversely for relative adjectives: the lack of such entailments is expected by the nullified culmination requirement. The question of partial and relative DA verbs in contexts that make a standard value explicit will not be discussed in this paper. ${ }^{7}$

\footnotetext{
${ }^{6}$ We can of course translate this assumption to frameworks that assume an event argument for the verb, by using the running time of the event as an indirect interval argument.

${ }^{7}$ In such contexts, the proposal in this paper expects an entailment from the DA verb to the positive form of the relative adjective, if the standard value remains constant through the entailment.
} 
total:

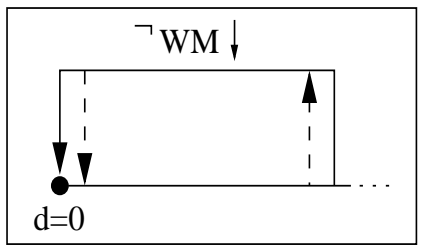

partial:

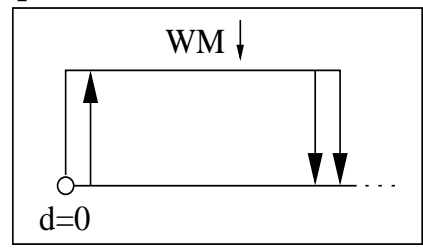

relative:

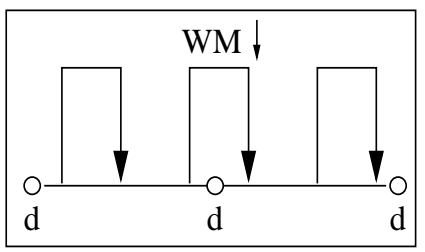

Figure 4: total, partial and relative DA verbs - solid (dashed) lines describe processes which are (are not, respectively) in the DA verb's denotation

Let us now formally define the A-V mapping using the progress and culmination requirements. To do that, we first assume that an adjective specifies degrees of entities over instants of time. Formally, this means that an adjective $A$ specifies a temporal dimension function $\operatorname{dim}_{A}$ that for each entity $x$ determines $x$ 's degree on the corresponding scale at any instant. Further, when a standard value is given, the adjective also characterizes which entities are associated with its positive form. This is done by deriving an open/closed set of degrees on the scale from the given standard value.

Definition 1 (adjectival information) Let $\left\langle S, \leq_{S}\right\rangle$ be a scale-a partially ordered set of degrees. An adjective that is associated with $\left\langle S, \leq_{S}\right\rangle$ specifies a function dim $_{A}$ and (optionally) a set of degrees $D_{A} \subseteq S$ such that:

$\operatorname{dim}_{A}:(E \times T) \rightarrow S$ is a temporal dimension function from entities in $E$ and temporal instants in $T$ to degrees in $S$;

$d \in S$ is an optional standard value in $S$, and $D_{A}=\left\{d^{\prime} \in S: d \leq_{S} d^{\prime}\right\}$ (an interval closed from below) or $D_{A}=\left\{d^{\prime} \in S: d<_{S} d^{\prime}\right\}$ (an interval open from below).

The intervals associated with a DA verb corresponding to an adjective $A$ are specified in the following definition using a function $V_{A}$ from entities in $E$ to sets of temporal intervals over $T$.

Definition 2 (A-V mapping) For an adjective $A$, let dim $_{A}$ be a temporal dimension function over a scale $\left\langle S, \leq_{S}\right\rangle$, and let $D_{A} \subseteq S$ be an optionally given set of degrees in $S$. Then the function $V_{A}$ maps every entity $x \in E$ to the set of intervals I over $T$, s.t. the following two conditions hold:

Progress: $\operatorname{dim}_{A}(x, \inf (I))<_{S} \operatorname{dim}_{A}(x, \sup (I))$;

Culmination: If $D_{A}$ is given, then $\operatorname{dim}_{A}(x, \sup (I)) \in D_{A}$.

For any adjective $A$, the function $V_{A}$ thus describes a binary relation between entities and temporal intervals, so that for every entity $x, V_{A}(x)$ is the set of intervals at which the DA verb corresponding to $A$ holds of $x$. For simplicity, we only treat

But (35) and (36) show that this does not have to be the case. I will not get here into the analysis of such effects, which may result from a change of standard value between the premise and conclusion of the entailment. 
here intransitive verbs, but the extension to verbs with more than one syntactic argument is straightforward. Standardly, the functions sup and inf correspond to the supremum and infimum functions, which here are necessary in order to deal with possibly open intervals.

\subsection{Weak Downward Monotonicity and (A)telicity}

Bennett and Partee (2003) provide a well-known definition of atelicity using what they call the subinterval property. Let $\mathscr{I}$ is the set of (open and closed) intervals over some partially ordered set. A set $\mathscr{J} \subseteq \mathscr{I}$ has the subinterval property iff it is downward monotone in $\mathscr{I}$. That is, for all $I, I^{\prime} \in \mathscr{I}$ s.t. $I^{\prime} \subseteq I \in \mathscr{J}: I^{\prime} \in \mathscr{J}$.

Bennett and Partee propose the subinterval property as the defining property of atelic predicates, and further assume that any predicate that lacks it is by definition telic. This proposal follows the intuition that atelic predicates like walk are 'homogeneous': if John walked between 2pm and 4pm, he obviously must have walked at many subintervals of the [2:00,4:00] interval, certainly in big enough subintervals. For instance, he must have walked between 2:01 and 3:59. With telic predicates, no subinterval property has to hold. For instance, if John drew a circle between $2 \mathrm{pm}$ and $4 \mathrm{pm}$, it is not necessary that there was any subinterval of [2:00,4:00] where he completed a circle, hence the lack of subinterval property.

A well-known problem with the subinterval property is that it puts too strong a restriction on atelic predicates. To be sure, if John walked between $2 \mathrm{pm}$ and $4 \mathrm{pm}$, it does not necessarily follow that he walked between 2:38 and 2:39. On that specific moment he might have stopped for a short rest without violating the truth of the premise. This problem for the subinterval definition of atelicity is reminiscent of the much discussed 'Sorites paradox' for the meaning of adjectives. For instance, supposing that a digital camera that costs $\$ 4,000$ is considered expensive, it reasonably follows that also a digital camera that costs $\$ 3,999$ is expensive. But it does not follow that a digital camera that costs $\$ 39$ is expensive. ${ }^{8}$

Thus, with both atelic predicates and adjectives that trigger the Sorites problem, some sort of downward monotonicity is required, but we cannot use the monotonic inference pattern 'all the way down'. I propose that what characterizes both situations is a notion that I call weak downward monotonicity $(W M \downarrow)$ : both the intervals associated with atelic predicates and the degrees associated with Soritessensitive adjectives allow approximation while going downward. Thus, from sentence (39a) we cannot draw conclusions about all subintervals of [2:00,4:00], but we can conclude the statement in (39b).

a. John walked between $2 \mathrm{pm}$ and $4 \mathrm{pm}$.

b. $\Rightarrow$ There is a proper subinterval $I^{\prime}$ of $[2: 00,4: 00]$ such that John walked in all the subintervals $I^{\prime \prime}$ that satisfy $I^{\prime} \subsetneq I^{\prime \prime} \subseteq[2: 00,4: 00]$.

\footnotetext{
${ }^{8}$ (Kennedy 2005) is a recent linguistic discussion of the Sorites problem that is especially relevant for our discussion, since Kennedy claims that only relative adjectives, but not partial or total adjectives, show the Sorites paradox.
} 
We can say that the interval $I^{\prime}$ approximates from below the interval [2:00,4:00] of John's walking. How much smaller than [2:00,4:00] intervals like $I^{\prime}$ are required to be is determined by context, but the requirement about at least one such $I^{\prime}$ is context-independent. This conception is inspired by the informal discussion of (Borik 2002), which I believe reflects a similar intuition. A typical situation that exemplifies this weak monotonicity requirement of atelic predicates is illustrated in Figure 5.

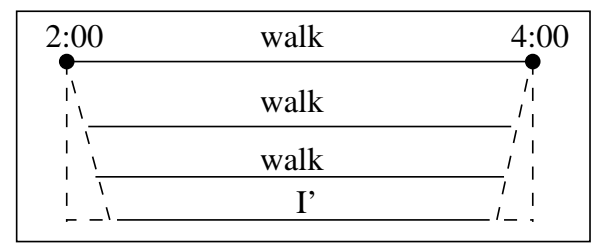

Figure 5: weak downward monotonicity with walk

I tentatively suggest that a similar approach can be used to deal with the Sorites problem in general. Thus, sentence (40a) does not entail that any specific price below $\$ 4,000$ is expensive for a digital camera; it only makes the requirement stated in (40b).

a. A digital camera that costs $\$ 4,000$ is expensive.

b. $\Rightarrow$ There is a price $p^{\prime}$ lower than $\$ 4,000$ such that for all prices $p^{\prime \prime}$ s.t. $p^{\prime}<p^{\prime \prime} \leq \$ 4,000$ : a camera that costs $p^{\prime \prime}$ is expensive.

How much lower than $\$ 4,000$ the price $p^{\prime}$ in (40b) is required to be is again determined by context.

Let us now formally define the notion of weak downward monotonicity. In this definition we say that an interval $I^{\prime}$ is properly within $I$ iff $\sup \left(I^{\prime}\right)<\sup (I)$ and $\inf \left(I^{\prime}\right)>\inf (I)$.

Definition 3 (weak downward monotonicity) Let $\mathscr{I}$ be the (open and closed) intervals over some partially ordered set. A set $\mathscr{J} \subseteq \mathscr{I}$ is weakly downward monotone $(W M \downarrow)$ iff for every $I \in \mathscr{J}$ there is $I^{\prime} \in \mathscr{I}$ s.t. $I^{\prime}$ is properly within $I$, and for every $I^{\prime \prime} \in \mathscr{I}:$ if $I^{\prime} \subsetneq I^{\prime \prime} \subseteq I$ then $I^{\prime \prime} \in \mathscr{J}$.

I propose the following hypothesis:

(41) For any atelic predicate $P$, the set of intervals associated with $P$ is $W M \downarrow$.

Thus, weak downward monotonicity is a necessary condition for atelicity. Hence, its absence is a sufficient condition for telicity. Weak monotonicity is only used here as a necessary condition for atelicity because predicates that pass standard tests for atelicity normally have stronger monotonicity requirements than weak downward monotonicity. For instance, in sentence (39), we would probably consider it an unnatural situation if the denotation of walk only contained intervals that approximate the two-hour interval [2:00,4:00], but no shorter intervals of walking. How to 
strengthen the hypothesis in (41) into a full definition of atelic denotations is something that I will not attempt to do here, especially since the hypothesis in (41) is sufficient for the purposes of this paper.

Using the proposed A-V mapping, we can apply hypothesis (41) to formally derive the (a)telicity of DA verbs. Note first that the A-V mapping makes a necessary requirement from DA verbs. However, this requirement itself does not yet guarantee that relative and partial DA verbs are atelic. This is as it should be, since we have already seen (cf. sentences (30), (31), (32)) that such DA verbs can show a telic behavior when context or world knowledge add a standard value that creates a closed interval. Furthermore, DA verbs like straighten, bend or widen do not necessarily reflect a gradual process in terms of the degree change on the adjective scale. And lack of graduality may block atelicity, as well as weak downward monotonicity. For instance, as Stephen Wechsler (p.c.) points out, when a road is widened very often no progress in its width happens before the final stages of the widening process. In such scenarios, the transitive verb widen may fail the progressive test for atelicity: if the workers are widening the road, it does not follow that they have widened it, even not a little.

We conclude that for a DA verb to satisfy weak downward monotonicity, and show atelic behavior, the intervals that it contains should be part of a gradual process along the scale, as described by the corresponding temporal dimension function. This notion of graduality is standard continuity and upward monotonicity of the temporal dimension function in its temporal argument, defined as follows.

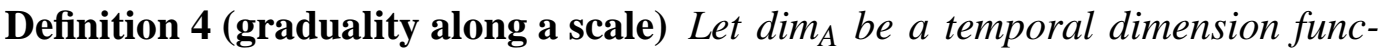
tion over a scale $\left\langle S, \leq_{S}\right\rangle$ for entities $E$ and temporal instants $T$. For $x \in E$, the function $\operatorname{dim}_{A}$ is said to be gradual along $\mathbf{S}$ in an interval $I \subseteq T$ iff $\lambda t$.dim $\operatorname{dim}_{A}(x, t)$ is upward monotonic in I, and for everyt in the closure of $I(=I \cup\{\inf (I)\} \cup\{\sup (I)\})$, for every $\varepsilon>0$ :

If $t>\inf (I)$ then there is $t^{\prime} \in I$ s.t. $t^{\prime}<t$ and $0 \leq \operatorname{dim}_{A}(x, t)-\operatorname{dim}_{A}\left(x, t^{\prime}\right)<\varepsilon$; If $t<\sup (I)$ then there is $t^{\prime} \in I$ s.t. $t<t^{\prime}$ and $0 \leq \operatorname{dim}_{A}\left(x, t^{\prime}\right)-\operatorname{dim}_{A}(x, t)<\varepsilon$.

Intuitively, a temporal dimension function $\operatorname{dim}_{A}$ describes a gradual process along the corresponding scale in an interval $I$ if $\operatorname{dim}_{A}$ describes progress along the scale in $I$ (i.e. $\operatorname{dim}_{A}$ is upward monotonic in $I$ ), and this progress in $I$ is continuous.

When a temporal dimension function describes a gradual process, our A-V mapping captures the parallelism between open/closed scales and $W M \downarrow / \neg W M \downarrow$ sets of intervals, respectively. The following proposition establishes weak downward monotonicity of the DA verbs corresponding to partial adjectives, whose scales are open, and to relative adjectives, where no standard value is given.

Proposition 1 Let $\operatorname{dim}_{A},\left\langle S, \leq_{S}\right\rangle, D_{A} \subseteq S$ and $V_{A}$ be as in definition 2. For some entity $x \in E$ assume that $\operatorname{dim}_{A}$ is gradual along $S$ in every $I \in V_{A}(x)$. If $D_{A} \subseteq S$ is given and open from below, or if $D_{A}$ is not given, then $V_{A}(x)$ is $W M \downarrow$.

The intuitive reason for this result is that graduality guarantees that progress in an interval $I$ entails progress in subintervals $I^{\prime}$ that approximate it. If $D_{A}$ is open 
from below and $I$ further attains a degree in $D_{A}$, the degree attained at the supremum of $I$ must be greater than the standard value. Graduality guarantees that this culmination at $I$ leads to culmination at intervals that approximate $I$ 's supremum. Both progress/culmination at $I$ thus lead to progress/culmination at intervals that approximate $I$ from below, hence the weak downward monotonicity of $V_{A}$. This is illustrated in Figure 4 above for partial and relative scales.

The following proposition shows that weak downward monotonicity is not guaranteed for total DA verbs, where the respective scale is closed from below.

Proposition 2 Let $\langle S, \leq S\rangle$ and $D_{A} \subseteq S$ be a scale and a set of values on it as in definition 2, where $D_{A}$ is closed from below. Then there is a temporal dimension function $\operatorname{dim}_{A}$ and an entity $x \in E$ s.t. $\operatorname{dim}_{A}$ is gradual for $x$ along $S$ for every $I \in$ $V_{A}(x)$, and $V_{A}(x)$ is not $W M \downarrow$.

Intuitively, the reason for this result is that, when $D_{A}$ is closed from below and an interval $I$ culminates at the infimum of $D_{A}$, even a gradual temporal dimension function cannot guarantee that any subinterval of $I$ would culminate within $D_{A}$. Hence $V_{A}$ is not $W M \downarrow$. This is illustrated in Figure 4 above for total scales.

Proposition 2 is weaker that what might be expected. It only guarantees telicity of total DA verbs in some cases, but not in all cases. This is sufficient at least in what concerns the main telicity criterion that is used in this paper: the lack of entailment involving the progressive. For the entailment to fail, it is enough to show one situation where the set of intervals associated with the verb is classified as telic (or $\neg W M \downarrow$ ). There are two reasons why the set of intervals that is associated with a total DA verb may not exhibit telicity. First, according to our definition, it may well happen that the temporal dimension function is gradual but not properly upward monotone. For instance, the children may clean the room gradually while at certain intervals within the process the cleanliness of the room remains constant. We have not excluded a situation where the process of cleaning the room ends with an interval with no progress in cleanliness. This may cause weak downward monotonicity. A second possible reason for weak monotonicity with total DA verbs, is that $\mathrm{R} \& \mathrm{~W}$ allow total adjectives like clean to be associated with negative degrees, in addition to the zero degree. $\mathrm{R} \& \mathrm{~W}$ do that in order to be able to compare in terms of cleanliness also entities that are categorized as clean. For instance, two tables can be called clean even though one of them is very clean or completely clean and the other one is not. If more than just the zero degree is allowed for total adjectives, a cleaning process may turn a clean object into a very clean object, while still satisfying both progress and culmination. This makes the process weakly downward monotone. In this paper I will not try to rule out such situations of atelic sets of intervals for total DA verbs, and I see no reason to consider them illicit. The distinction between total DA verbs and partial/relative DA verbs in terms of telicity is captured by the current assumptions above, and we do not need to strengthen these assumptions without further motivation. 


\section{Back to Path Structures}

In the previous section we have seen a parallelism between the closure of a scale and the weak downward monotonicity of the corresponding DA verb. However, the starting point of this paper was that the behavior of spatial prepositions with almost suggests that the parallelism between closure and telicity is not complete. This section briefly proposes an account of the opposition between the case of DA verbs and the case of directional prepositions. Reconsider the contrast between directional prepositions like to in English, and prepositions like @ ad in Hebrew or tot in Dutch. To account for the contrasts in acceptability of almost modification, I proposed that the former preposition describes an open path whereas the latter two prepositions describe closed paths. Both kinds of preposition give rise to telic predicates when they combine with verbs. To see why, we need to focus on the semantics of verb phrases with such directional prepositions.

Let us refer by TO to prepositions of the to variety and by TOT to prepositions of the @ad/tot variety. Following many works (see e.g. Zwarts and Winter (2000) and the references therein), we assume that the denotation of a directional preposition is a collection of spatial paths: functions from intervals to locations in space. Let $\mathscr{I}$ be the set of intervals and $L$ be the set of locations, described using our favorite spatial ontology. The denotations of PPs like TO the park or TOT the park are collections of functions whose domains are (possibly different) intervals in $\mathscr{I}$ and their ranges are $L$. Specifically, we assume:

TO_. $\mathbf{p}^{\prime}=\bigcup_{I \in \mathscr{I}}\left\{f \in L^{I}: I\right.$ is open from above and $\left.f(\sup (I))=\operatorname{loc}\left(\mathbf{p}^{\prime}\right)\right\}$

TOT_ $\mathbf{p}^{\prime}=\bigcup_{I \in \mathscr{I}}\left\{f \in L^{I}: I\right.$ is closed from above and $\left.f(\max (I))=\operatorname{loc}\left(\mathbf{p}^{\prime}\right)\right\}$

Here we assume that $\operatorname{loc}\left(\mathbf{p}^{\prime}\right)$ is the (static) location of the park. The difference between the two collections of paths is that in the first case, the path functions are from open intervals whereas in the second case these are closed intervals. In both cases, at the supremum of the interval the location attained by the path is the location of the park. In the case of TOT, this supremum is moreover a maximum.

Verb phrases like ran or ran TO/TOT the park are associated with a set of intervals for each entity argument. The latter modified VPs are derived from the verb (or simple VP) ran by first looking at the location of the subject in each instant. Let us use a temporal location function for this purpose, such that $l o c(x, t) \in L$ is the location of an entity $x \in E$ at an instant $t \in T$. To form the denotation of a VP of the form [V PP], those intervals for which the loc function describes a path in the denotation of PP are intersected with the intervals of V. Formally:

$$
\llbracket \mathrm{V} \mathrm{PP} \rrbracket(x) \stackrel{\text { def }}{=} \llbracket \mathrm{V} \rrbracket(x) \cap\left\{I \in \mathscr{I}:\left(\lambda t_{t \in I} \cdot \operatorname{loc}(x, t)\right) \in \llbracket \mathrm{PP} \rrbracket\right\}
$$

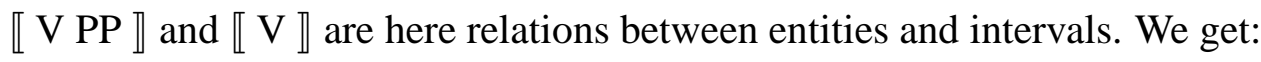

$$
\llbracket \text { run TO the park } \rrbracket(x)=\operatorname{run}^{\prime}(x) \cap\left\{I \in \mathscr{I}:\left(\lambda t_{t \in I} . \operatorname{loc}(x, t)\right) \in \text { TO_} \mathbf{p}^{\prime}\right\}
$$

In words: the intervals where $x$ ran TO the park are those intervals where $x$ ran, and where $x$ 's change of location created a path TO the park. By assumption on TO, (43) is equal to: 
$\operatorname{run}^{\prime}(x) \cap\left\{I \in \mathscr{I}: I\right.$ is open from above and $\left.\operatorname{loc}(x, \sup (I))=\operatorname{loc}\left(\mathbf{p}^{\prime}\right)\right\}$.

In words: $I$ is an interval at which $x$ ran TO the park if $I$ is open from above and at I's upper limit $x$ was at the park. Further, consider TOT:

$$
\begin{aligned}
& \llbracket \text { run тот the park } \rrbracket(x)=\operatorname{run}^{\prime}(x) \cap\left\{I \in \mathscr{I}:\left(\lambda t_{t \in I} \cdot \operatorname{loc}(x, t)\right) \in \text { TOT_} \mathbf{p}^{\prime}\right\} \\
& =\operatorname{run}^{\prime}(x) \cap\left\{I \in \mathscr{I}: I \text { is closed from above and } \operatorname{loc}(x, \max (I))=\operatorname{loc}\left(\mathbf{p}^{\prime}\right)\right\}
\end{aligned}
$$

In words: $I$ is an interval at which $x$ ran TOT the park if $I$ is closed from above and at the end of $I, x$ was at the park.

The difference we argued for concerning the acceptability of almost modification of (43) (marginal) and (44) (OK), follows from the open intervals in (43) as opposed to the closed intervals in (44). Crucially, however, both sets of intervals are not necessarily $W M \downarrow$. With respect to both TO and TOT, intervals at the end of which $x$ was at the park do not necessarily contain any smaller interval at the end of which $x$ was at the park. In particular this is true when $x$ 's motion, described using the temporal location function, is 'smooth' or 'gradual' in a sense parallel to the definition of graduality of temporal dimension functions.

We conclude that open path structures, as opposed to open scalar structures, do not necessarily involve an atelic temporal-aspectual behavior. Whether an open structure creates atelicity depends on the mapping from this structure to the temporal-aspectual structure of verb. Specifically, the A-V mapping derives atelic $(W M \downarrow)$ structures from open structures, whereas the P-V mapping does not. This contrast is illustrated in Figure 6, where $I^{\prime}{ }_{1} \subseteq I$ illustrates weak downward monotonicity with an open scale of the partial DA verb bend, but $I_{2}^{\prime} \subseteq I$ illustrates lack of weak downward monotonicity with the open path of the TO preposition.

In Section 4 we saw that closed scales lead to telicity. Similar considerations can now be used to show that closed paths lead to telicity as well. In Section 4 we also saw that due to the definition of the A-V mapping, open scales furthermore lead to atelicity of DA verbs. We have now seen that this is no longer the case for the $\mathrm{P}-\mathrm{V}$ mapping, where open path structures can appear with telic prepositions. This accounts for the incomplete correlation between telicity and almost modification with PP constructions: while S-interpretations of almost are impossible with atelic prepositions, their availability with telic prepositions is restricted by closure.

bend:

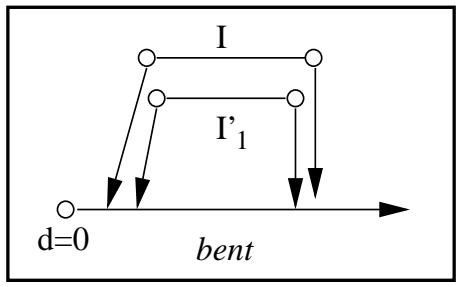

walk to the park:



Figure 6: weak downward monotonicity of bend, as opposed to walk in the parkin both cases $I$ is associated with the predicate; however $I_{1}^{\prime}$ is associated with bend whereas $I_{2}^{\prime}$ is not associated with walk to the park 


\section{References}

Amaral, P.: 2006, 'On the Semantics of Almost'. Paper presented at the LSA. January 2006.

Bennett, M. and B. Partee: 2003, 'Toward the Logic of Tense and Aspect in English', in B. Partee (ed.), Compositionality in Formal Semantics: Selected Papers of Barbara Partee. Blackwell, Oxford. Reprinted from an unpublished 1972 article, distributed in 1978 by Indiana University Linguistics Club.

Borik, O.: 2002, Aspect and Reference Time, PhD dissertation, Utrecht University. Cruse, D. A.: 1986, Lexical Semantics. Cambridge University Press, Cambridge.

Declerck, R.: 1979, 'Aspect and the Bounded/Unbounded (Telic/Atelic) Distinction', Linguistics 17, 761-794.

Dowty, D.: 1979, Word Meaning and Montague Grammar: the Semantics of Verbs and Times in Generative Semantics and in Montague's PTQ. D. Reidel, Dordrecht.

Frazier, L., C. Clifton Jr., and B. Stolterfoht: 2006, 'Scale Structure: Processing Minimum Standard and Maximum Standard Scalar Adjectives'. Unpublished ms., University of Massachusetts at Amherst.

Hay, J., C. Kennedy, and B. Levin: 1999, 'Scalar Structure Underlies Telicity in 'Degree Achievements", in Proceedings of SALT IX. CLC Publications, Cornell, Ithaca, NY.

Kearns, K.: 2005, 'Telic Senses of Deadjectival Verbs'. To appear in Lingua.

Kennedy, C.: 2005, 'Vagueness and Grammar: the Semantics of Relative and Absolute Gradable Predicates'. Unpublished ms., University of Chicago. To appear in Linguistics and Philosophy.

Kennedy, C. and L. McNally: 2005, 'Scale Structure and the Semantic Typology of Gradable Predicates', Language 81, 345-381.

Rapp, I. and A. von Stechow: 1999, 'Fast 'Almost' and the Visibility Parameter for Functional Adverbs', Journal of Semantics 16.2, 149-204.

Rotstein, C. and Y. Winter: 2004, 'Total Adjectives vs. Partial Adjectives: Scale Structure and Higher-Order Modification', Natural Language Semantics 12, 259-288.

Yoon, Y.: 1996, 'Total and Partial Predicates and the Weak and Strong Interpretations', Natural Language Semantics 4, 217-236.

Zwarts, J.: 2005, 'Prepositional Aspect and the Algebra of Paths', Linguistics and Philosophy 28.6, 739-779.

Zwarts, J. and Y. Winter: 2000, 'Vector Space Semantics: a Model-Theoretic Analysis of Locative Prepositions', Journal of Logic, Language and Information 9, 169-211. 